\title{
Prognostic value of bone marker beta-crosslaps in patients with breast carcinoma
}

\author{
Nicole Zulauf ${ }^{1}$, Ingo Marzi ${ }^{2}$ and Gerhard M Oremek ${ }^{1 *}$ \\ ${ }^{1}$ Department of Laboratory Medicine, Johann Wolfgang Goethe University, Frankfurt/Main, Germany \\ ${ }^{2}$ Department of Surgery, Johann Wolfgang Goethe University, Frankfurt/Main, Germany
}

\begin{abstract}
This study investigated the usefulness of bone markers Alkaline Phosphatase (AP) and Beta-Crosslaps (ß-CTx) to diagnose, treat and monitor patients with breast carcinoma. AP is a marker of bone formation, while $\beta-C T x$ are markers of bone resorption. $ß-C T x$ are expressed as degradation products of collagen type I and can be measured in blood and urine. The aim of this project was to evaluate the significance of bone markers $ß-\mathrm{CTx}$ and AP with regard to their usage for early diagnostics of bone metastases and pathological bone metabolism in pre- and postmenopausal patients with known breast cancer. Peripheral blood samples of patients with mammarial diseases (benign and malign) were collected and the bone markers AP and $B-C T x$ determined. A total number of 110 patients had Benign Mammarial Diseases, BMD (30 fibroadenoma, 50 mastopathy and 30 hypertrophy patients). 30 patients were suffering from a malignant breast cancer without bone metastases. 50 patients had known bone metastases. The determination of B-CTx was conducted based on the Immunoassay "ECLIA", the analysis approach Elecsys 2010 and cobas e by Roche Diagnostics (Mannheim, Germany). The analysis of AP was performed with the HYDRAGEL ISO-PAL kits and HYDRASYS electrophoresis system of Sebia (Fulda, Germany). For the detection of bone metastases, the study showed a sensitivity of $94.0 \%$ with $86.67 \%$ specificity for AP. Results for $ß-\mathrm{CTx}$ had a sensitivity and specificity of $100.0 \%$. An elevated $\beta-\mathrm{CTx}$ activity was associated significantly with bone metastases in the study groups ( $\mathrm{p}=0.000000$ ). Furthermore there were significant differences $(\mathrm{p}=0.000000)$ due to the menopausal status of patients. In conclusion $\beta_{-}-\mathrm{CT} \mathrm{x}$ are more sensitive and specific to detect bone metastases and bone turnover than AP. In patients with multimorbidity the origin of AP is not clear due to its multi-organic appearance. Hence $3-\mathrm{CTx}$ are considered to be helpful in the diagnostic procedure of breast cancer-patients to detect bone metastases. Moreover they can be utilized to indicate patients with early dysfunctions in bone metabolism and allow inducing early treatment. ß-CTx as indicators of bone resorption, are capable to provide a significant differentiation between mamma-carcinoma patients with and without bone affection.
\end{abstract}

\section{Introduction}

Breast cancer and metastases induced by this disease are topics of medical research through decades. Bone is a typical location for metastases which often increase pain, morbidity and mortality. Through the past years several laboratory methods and markers have been developed to assist the diagnosis of bone lesions. The accurate diagnostic of bone metastases and early induced antiresorptive/cancer therapies are essential to reduce morbidity and mortality.

Bone is a tissue which underlies continuous resorption and formation. Malignant tumors which spread to bone are able to cause osteolytic, osteoblastic or mixed lesions. Bone is resorbed by osteoclasts rather than through the tumor cells [1-4]. Under these circumstances bone turnover is elevated and some of the involved enzymes and metabolic products can be measured.

Several markers of bone turnover with different sensitivity and specificity values have already been described in various studies. Alkaline phosphatase is one marker of bone formation which is often used due to its common availability in laboratory analysis [5,6]. It is a membrane-bound enzyme which can be found in diverse tissues of the body, such as bone, liver, kidney and intestine. Though the exact function in bone metabolism is unknown, AP increases when mineralization of bone is in progress $[7,8]$.

In a healthy adult $50 \%$ of the total amount of AP are originated from liver and $50 \%$ from bone. In children and growing infants up to $90 \%$ of the total amount is of bone origin due to the higher expansive bone metabolism. If liver disease is ruled out, AP provides an overview of the osteoblastic activity [9-14].

The B-CTx are fragments of collagen type I, which are released during bone resorption [15]. These C-terminal cross-linking telopeptides of collagen type I are specific fractions of C-terminal end of collagen, which are directly abounded at the beginning of collagen depletion [16]. They are detected in blood via specific antibodies. With increasing bone degradation an elevated amount of these segments can be verified in blood [15]. A correlation between B-CTx and other markers of bone turnover or bone mineral density have also been observed [17-19]. As the C-terminal telopeptide of collagen type I, its usefulness as a marker of bone turnover has been described earlier, especially in osteoporosis management [20]. Although these markers are potential instruments for the diagnostic of bone metastases, further

Correspondence to: Gerhard M. Oremek, Department of Laboratory Medicine, Internal Medicine, Hospital of the Johann Wolfgang Goethe University, Theodor Stern Kai 7 , D-60590 Frankfurt/ Main, Germany, Tel: +49(0)69/ 63015024/7823, E-mail: Gerhard-Maximilian.Oremek@kgu.de

Key words: Bone marker, tumor marker, bone metastases, malignancy, alkaline phosphatase, beta-crosslaps, CA 15-3, breast cancer, breast carcinoma, mammarial diseases

Received: September 24, 2014; Accepted: September 30, 2014; Published: October 03, 2014 
analysis has been recommended to confirm their prediction to diagnose bone turnover and monitor antiresorptive treatment.

The aim of this study was to evaluate the clinical value of AP and $ß-C T x$ to detect bone metastases and pathological bone metabolism in a collective of different patients with diagnosed breast cancer. To achieve comparable results, the clinically well-established bone marker AP in combination with further special tumor markers (CA 15-3 and partially CEA) were evaluated and compared with respect to bone metabolism.

\section{Materials and methods}

The total of 190 serum samples which were collected within one year by the gynaecological department of JWG University Clinic Frankfurt a. M. were analyzed with regard to AP, CA 15-3 and B-CTx and divided into following populations to allow a differentiated evaluation, comparison and conclusion: 110 patients with benign mammarial diseases (BMD: 30 Fibroadenoma, 50 Mastopathy and 30 Hypertrophy patients), 80 patients with breast cancer (30 without and 50 with bone metastases). Furthermore additional 200 proband samples were leveraged for the determination of reference values for ß-CTx as well as for CA 15-3 (50 pre- and 50 postmenopausal healthy women for each marker). These subjects (consent presumed) were selected into mentioned populations during their hospitalization in gynaecological department of JWG University Clinic Frankfurt a. M. concerning their respective diagnoses. Due to exact knowledge of the particular diagnosis, participants were allocated to appropriate groups instead of random selection (open-label study).

The analysis of AP was performed with HYDRAGEL ISO-PAL kits and HYDRASYS electrophoresis system of Sebia (Fulda, Germany). The determination of $\mathrm{B}$-CTx was conducted based on the Immunoassay "ECLIA" and the analysis approach Elecsys 2010 and cobas e by Roche Diagnostics GmbH (Mannheim, Germany) with two specific monoclonal antibodies. After incubation of the antigen of sera samples with biotinylated-antibody and ruthenium-conjugated-antibody a sandwich-complex is assembled. This complex is fixed on the solid phase via the assignment of streptavidin-coated microparticles. Finally the resulting product can be measured with photomultiplier and electrochemiluminescence.

The colorimetric measurement to determine B-CTx activity was executed with the ElectroChemiLuminescenceImmunoAssay, ECLIA on Elecsys and cobas e systems (Roche Diagnostics GmbH, Germany).

\section{Statistical analysis}

All the statistical calculations were operated with SPSS Statistics 21.0, Microsoft Word 2007 and Microsoft Excel 2007.

\section{Results}

The results of AP and B-CTx activity in the different study groups are summarized in Table 1.
The mean activity of AP in the group without malignancy was $134,43 \mathrm{ng} / \mathrm{ml}$ (median: 135,0 ng/ml), in patients with malignant disease but without bone metastases 129,1333 ng/ml (median: 115,0 ng/ml) and in patients with bone metastases $284,24 \mathrm{ng} / \mathrm{ml}$ (median: 277,0 ng/ $\mathrm{ml})$.

The serum levels of $\beta$-CTx activity in patients without malignancy showed a mean of $0,4521 \mathrm{ng} / \mathrm{ml}$ (median: $0,32 \mathrm{ng} / \mathrm{ml}$ ). The highest activities were found in patients with malignancy and additional bone metastases (mean: 75,382 ng/ml, median: $67,0 \mathrm{ng} / \mathrm{ml}$ ) and in patients with malignancy (mean activity of 0,893 ng/ml, median: 0,905 ng/ml).

Bone-metastases-positive patients offered significantly higher $ß$-CTx serum concentration $(p=0,000000)$ compared to the bonemetastases-negative collectives. Moreover there were significant differences observed within the standard values $(p=0,000000)$ related to the menopausal status with tendency to higher values in postmenopause. Among the benign mammarial diseases pathological elevated results were identified for fibro adenomas with noevidence for any malign process. No significant difference compared to patients with carcinoma without skeletal affection was shown in contrast to the other two BMD populations $(p=0,820069)$. For diagnostics of osseous metastases, B-CTx achieved a sensitivity and specificity of $100,0 \%$. By comparing the covered areas (AUC) of Receiver Operating Characteristic Curves (ROC) the very positive diagnostic performance of 1,0 of $B$-CTx was carved out. This outperformed CA 15-3, which represented results of 0,960667 as well as AP with results of 0,940333 . Demonstrated on the basis of a patient example (therapeutic conditions: operation and chemotherapy) the serum concentration of $ß-C T x$ declined $(175,8 \mathrm{ng} /$ $\mathrm{ml}$ to $80,4 \mathrm{ng} / \mathrm{ml})$, though it increased again massively in case of relapse $(199,4 \mathrm{ng} / \mathrm{ml})$.

A significant difference between the patients without malignant diseases, those with malignancy and the patients with additional bone metastases was therefore be pointed for AP ( $p=0,000060$ and 0,000000 ) and $B-C T x(p=0,000000)$.

The distribution of AP and $\mathrm{B}-\mathrm{CTx}$ in the gynaecological patients is posed in Figure 1 and 2.

Figure 3 and 4 indicate the categorized results of AP and B-CTx whether they were normal or above normal activity. For both bone markers a significant difference in the different study groups $(\mathrm{p}=0,000000)$ was figured out. We found a significant association between the occurrence of bone metastases and elevated parameter activity in the study.

For the detection of bone metastases the sensitivity of AP was $94,0 \%$ and for $B$-CTx $100,0 \%$ with a specificity for AP of $86,67 \%$ and for $\beta$-CTx of $100,0 \%$.

\section{Discussion}

In the last years several different bone markers have been investigated to assist the diagnostic of bone turnover. However the

Table 1. Activity of $\beta-C T x$ and AP $[\mathrm{ng} / \mathrm{ml}]$ in the three different study groups.

\begin{tabular}{|c|c|c|c|c|c|c|c|}
\hline & & & B-CTx [ng/ml] & & & AP [ng/ml] & \\
\hline & $\mathbf{N}$ & Mean & Mean \pm 2SD & Range & Mean & Mean \pm 2SD & Range \\
\hline Patients without malignant disease & 110 & 0,4521 & $0,4521 \pm 0,0564$ & $0,11-1,21$ & 134,43 & $134,43 \pm 1,9728$ & $117,0-150,0$ \\
\hline $\begin{array}{l}\text { Patients with known breast cancer without bone } \\
\text { metastasis }\end{array}$ & 30 & 0,893 & $0,893 \pm 0,0554$ & $0,58-1,2$ & 129,1333 & $129,1333 \pm 16,484$ & $92,0-270,0$ \\
\hline $\begin{array}{l}\text { Patients with known breast cancer with bone } \\
\text { metastasis }\end{array}$ & 50 & 75,382 & $75,382 \pm 14,677$ & $12,4-318$ & 284,24 & $284,24 \pm 31,2246$ & $114,0-682,0$ \\
\hline
\end{tabular}




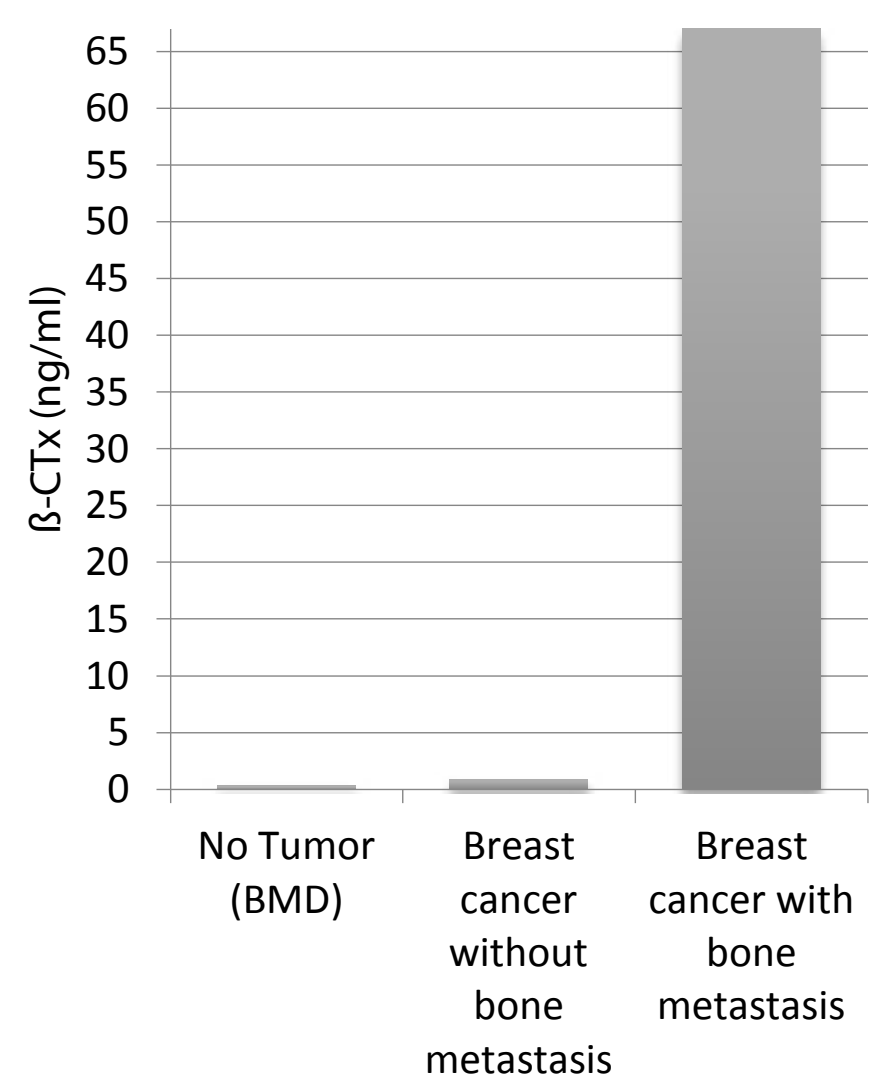

Figure 1. Distribution of median $\beta-C T x$ in the study.

Activityof $\beta-C T x(n g / m l)$ against patients without a malignant disease (BMD), patients with breast cancer but without bone metastases, patients with breast cancer and bone metastases.

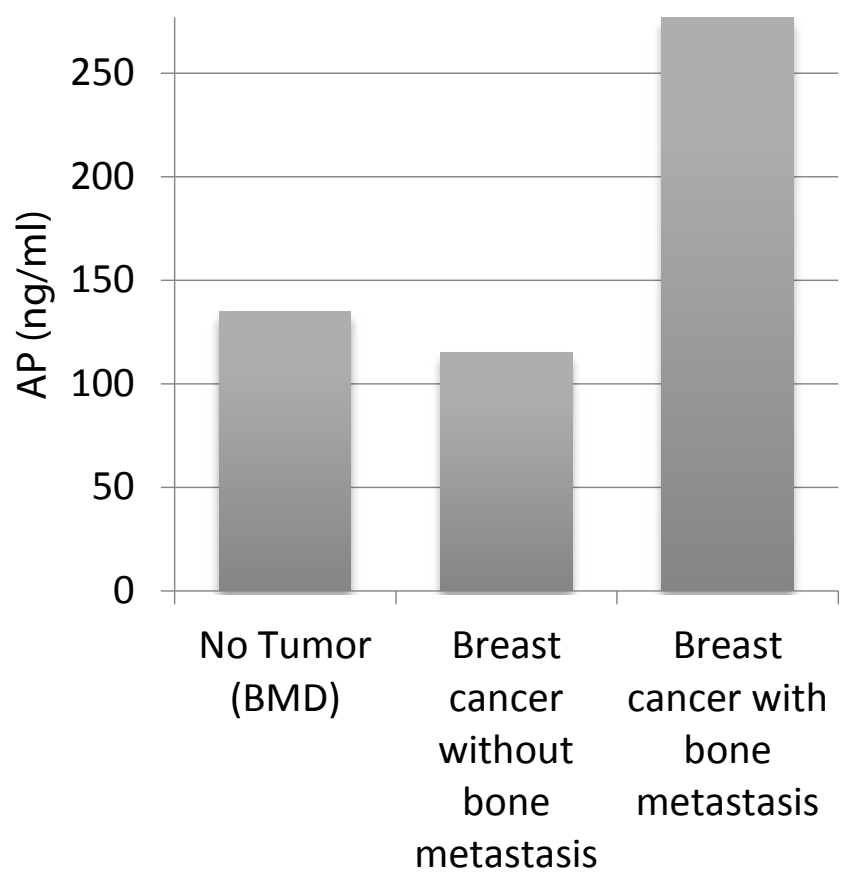

Figure 2. Distribution of median AP in the study.

Activity ofAP $(\mathrm{ng} / \mathrm{ml})$ against patients with BMD, patients with breast cancer but without bone metastases, patients with breast cancer and bone metastases. accuracy of those markers does not allow being the only diagnostic instrument to predict the appearance of bone metastases. So far none of the known markers could be solely used as screening parameters for patients to diagnose metastases to bone.

The measured sensitivity and specificity for AP and B-CTx to detect bone metastases in our study are partially contrary to results of earlier investigations: for example the sensitivity of $\mathrm{AP}$ is $67,0 \%$ and the specificity $100,0 \%$, whereas $B$-CTx are not able to verify the presence of bone metastases or the sensitivity/specificity of $B$-CTx is only $53,0 \%$ / $45,0 \%$ and concisely lesser than our results demonstrate [21-24].

The slightly poorer sensitivity and specificity for AP in comparison to ß-CTx appear to be caused by the multimorbidity of our patients and the known composition of total-AP by different isoenzymes.

No one of our patients with known malignancy and bone metastases show pathological elevated serum levels of $B-C T x$ (Figure 3). As investigated before, we find a very strong (spearman coefficient of correlation: 0,8355 ) and high positive significant correlation on level $\alpha=0,05$ between our measurements of AP and $\beta-C T x$ relating to breast carcinoma-patients [19].

In comparison to $\mathrm{AP}$, the $\mathrm{B}$-CTx are the more specific bone markers to detect bone metastases in our study. Namely they outperform parameters established in today's routines by providing improved sensitivity and specificity. Furthermore they exhibit adequate characteristics to monitor ongoing therapies. Based on the detected values it is possible to determine a specific therapy's success and to monitor its progress accordingly. A significant correlation between the activity of $\beta$-CTx and the occurrence of bone metastases

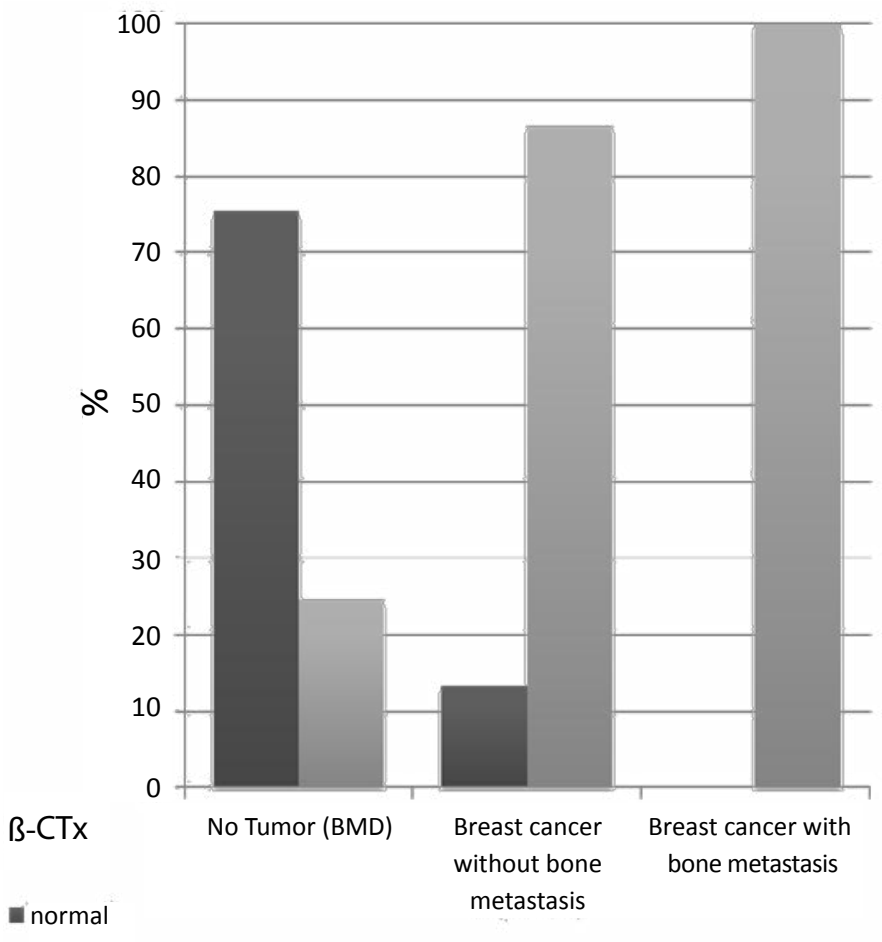

Elevated

Figure 3. Distribution of $\beta-C T x$ in the study groups.

Inpatients with BMD (no tumor) $75,45 \%$ were of normal activity, $24,55 \%$ were pathological elevated. Patients with tumor but without bone metastases showed normal activity in $13,33 \%$ and elevated levels of $\beta-C T x$ in $86,67 \%$. The $\beta-C T x$ levels were normal in $0,0 \%$ of all patients with known bone metastases and elevated in $100,0 \%$. 


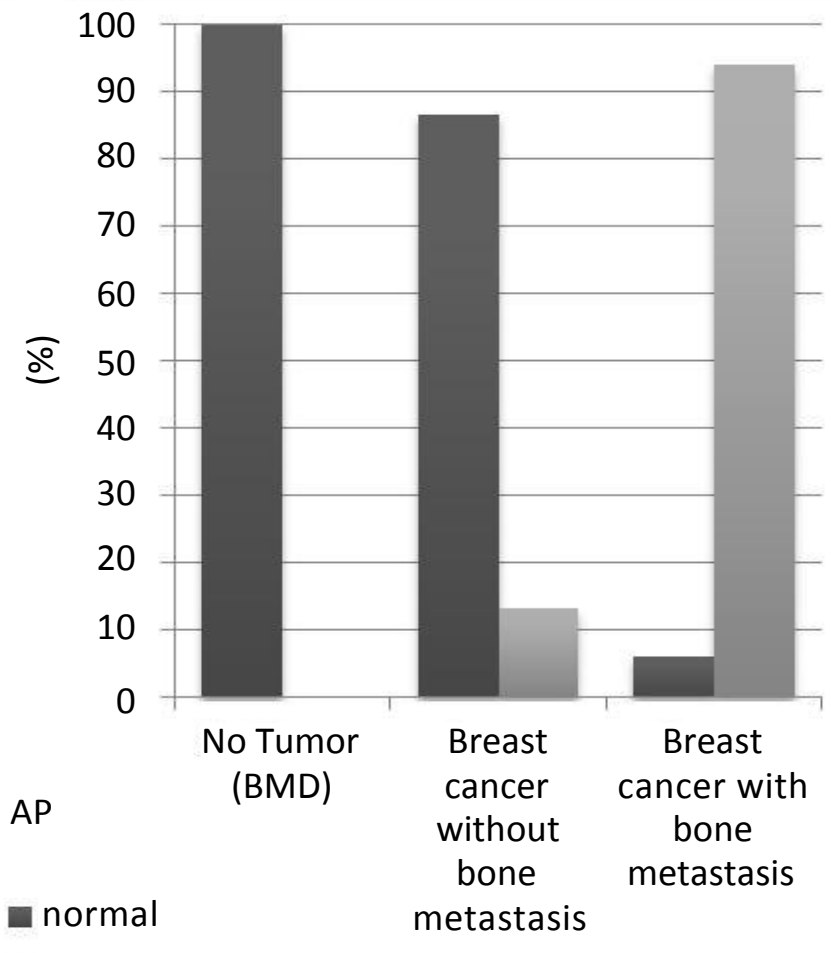

- elevated

Figure 4. Distribution of AP in the study groups.

In patientswith BMD (no tumor) $100,0 \%$ were of normal activity, $0,0 \%$ were pathological elevated. Patients with tumor but without bone metastases showed normal activity in $86,67 \%$ and elevated levels of AP in $13,33 \%$. The AP levels were normal in $6 \%$ of all patients with known bone metastases and elevated in $94 \%$.

is statistically proved $(\mathrm{p}=0,000000)$. In addition $ß-\mathrm{CTx}$ show a significant rise in postmenopausal patients $(p=0,000000)$ in our study. It is known that endocrinological changes affect the bone metabolism and osteoporosis with bone degradation occurs more often. Due to increased postmenopausal occurrence of osteoporosis, the inherent accelerated bone resorption and increased ß-CTx-concentrations, for the diagnostic of bone metastases higher cutoff-values should be considered for the postmenopausal women compared to the collective of men and premenopausal women.

We suggest a further investigation and evaluation of B-CTx as markers of bone turnover. They seem to be helpful diagnostic parameters of pathological elevated bone metabolism and should be used in follow up examinations of breast cancer-patients. Moreover they appear not to be only a beneficial instrument to get an idea of the bone metabolism, but may be also profitable to assist an antiresorptive treatment in time to those patients withprogressive affection of bone [25-27].

\section{References}

1. Boyce BF, Yoneda T, Guise TA (1999) Factors regulating the growth of metastatic cancer in bone. Endocr Relat Cancer 6: 333-347. [Crossref]

2. Pollari S, Leivonen SK, Perälä M, Fey V, Käkönen SM, et al. (2012) Identification of microRNAs inhibiting TGF- $\beta$-induced IL-11 production in bone metastatic breast cancer cells. PLoS One 7: e37361. [Crossref]

3. Mundy GR, Martin TJ (1993) Pathophysiology of skeletal complications of cancer. Physiology and Pharmacology of Bone 107: 641-671.
4. Clézardin P (2013) [Pathophysiology of bone metastases and new molecular targets involved in bone remodelling]. Bull Cancer 100: 1083-1091. [Crossref]

5. Oremek GM, Weis A, Sapoutzis N, Sauer-Eppel H (2003) Diagnostic value of bone and tumour markers in patients with malignant diseases. Anticancer Res 23: 987-990. [Crossref]

6. Molina R, Bosch X, Auge JM, Filella X, Escudero JM, et al. (2012) Utility of serum tumor markers as an aid in the differential diagnosis of patients with clinical suspicion of cancer and in patients with cancer of unknown primary site. Tumour Biol 33: 463-474. [Crossref]

7. Lian JB, Stein GS, Aubin JE (2003) Anatomy and Biology of Bone Matrix and Cellular Elements. Bone Formation: Maturation and Functional Activities of Osteoblast Lineage Cells. American Society for Bone and Mineral Research, Primer, Section 1: 13-27.

8. Mackiewicz Z, Niklinska WE, Kowalewska J, Chyczewski L (2011) Bone as a source of organism vitality and regeneration. Folia Histochem Cytobiol 49: 558-569. [Crossref]

9. Fohr B, Dunstan CR, Seibel MJ (2003) Clinical review 165: Markers of bone remodeling in metastatic bone disease. J Clin Endocrinol Metab 88: 5059-5075. [Crossref]

10. Debiais F (2013) [Biomarkers of bone remodelling]. Bull Cancer 100: 1093 1100. [Crossref]

11. Woitge HW, Seibel MJ (2001) Biochemical markers to survey bone turnover. Rheum Dis Clin North Am 27: 49-80. [Crossref]

12. Galliera E, Luzzati A, Perrucchini G, Gagliano F, Colloredo Mels L, et al. (2012) Bone formation and resorption markers as diagnostic tools for bone metastases evaluation. Int J Biol Markers 27: e395-399. [Crossref]

13. Mose S, Menzel C, Kurth AA, Obert K, Breidert I, et al. (2003) Tartrate-resistant acid phosphatase $5 \mathrm{~b}$ as serum marker of bone metabolism in cancer patients. Anticancer Res 23: 2783-2788. [Crossref]

14. Hung MJ, Oremek GM (2011) Value of TRAcP 5b as a diagnostic marker for detection of bone metastases in patients with breast cancer. Eur J Gynaecol Oncol 32: 615-618. [Crossref]

15. Lehmann M, Geissel W, Kreutz I, Ott C, Overbeck P, et al. (2008) Osteoporose: KnochenabbaulässtsichmitBlutmarker Beta-Crosslapsbestimmen. Biomarker belegtKnochenschwund. Test auf Abbauprodukte des Knochenkollagens/ Mö glichkeitzurÜberwachungeinerOsteoporose-Therapie. ÄrzteZeitungVerlagsGmbH,Springer, Neu-Isenburg.

16. Lippuner K, Johansson H, Kanis JA, Rizzoli R (2010) FRAX assessment of osteoporotic fracture probability in Switzerland. Osteoporos Int 21: 381-389. [Crossref]

17. Body JJ, Dumon JC, Gineyts E, Delmas PD (1997) Comparative evaluation of markers of bone resorption in patients with breast cancer-induced osteolysis before and after bisphosphonate therapy. Br J Cancer 75: 408-412. [Crossref]

18. Lipton A, Cook R, Brown J, Body JJ, Smith M, et al. (2013) Skeletal-related events and clinical outcomes in patients with bone metastases and normal levels of osteolysis: exploratory analyses. Clin Oncol (R Coll Radiol) 25: 217-226. [Crossref]

19. Kanakis I, Nikolaou M, Pectasides D, Kiamouris C, Karamanos NK (2004) Determination and biological relevance of serum cross-linked type I collagen $\mathrm{N}$-telopeptide and bone-specific alkaline phosphatase in breast metastatic cancer. J Pharm Biomed Anal 34: 827-832. [Crossref]

20. Wheater G, Elshahaly M, Tuck SP, Datta HK, van Laar JM (2013) The clinical utility of bone marker measurements in osteoporosis. J Transl Med 11: 201. [Crossref]

21. Seregni E, Martinetti A, Ferrari L, Bombardieri E (2001) Clinical utility of biochemical marker of bone remodelling in patients with bone metastases of solid tumors. QJ Nucl Med 45: 7-17. [Crossref]

22. Coleman R, Brown J, Terpos E, Lipton A, Smith MR, et al. (2008) Bone markers and their prognostic value in metastatic bone disease: clinical evidence and future directions. Cancer Treat Rev 34: 629-639. [Crossref] 
23. Le Bricon T, Gay-Bellile C, Cottu P, Benlakehal M, Guillon H, et al. (2010) Lectin affinity electrophoresis of serum alkaline phosphatase in metastasized breast cancer. J Clin Lab Anal 24: 20-24. [Crossref]

24. Pollmann D1, Siepmann S, Geppert R, Wernecke KD, Possinger K, et al. (2007) The amino- terminal propeptide (PINP) of type I collagen is a clinically valid indicator of bone turnover and extent of metastatic spread in osseous metastatic breast cancer. Anticancer Res 27: 1853-1862. [Crossref]
25. Koizumi M, Takahashi S, Ogata E (2003) Bone metabolic markers in bisphosphonate therapy for skeletal

26. Zulauf N, Speda MD, Oremek GM, Marzi I (2013) Prognostic value of bone markers in patients with carcinoma. J Mol Biomark Diagn 5: 159.

27. Klitscher D, Herrmann M, Frank J, Marzi I, Herrmann W, et al. (2012) Changes in markers of bone metabolism following surgery. Recent patents on biomarkers 2: $60-68$.

Copyright: (C2014 Zulauf N. This is an open-access article distributed under the terms of the Creative Commons Attribution License, which permits unrestricted use, distribution, and reproduction in any medium, provided the original author and source are credited. 